\title{
How does familiarity affect visual search for letter strings?
}

\author{
JOHN H. FLOWERS and DORIS J. LOHR \\ University of Nebraska, Lincoln, Nebraska
}

\begin{abstract}
Subjects visually searched for letter string targets consisting of either familiar English threeletter words (e.g., SEX) or featurally similar nonword trigrams (e.g., SFX). Distractor items were either words or nonwords and had varying degrees of feature overlap with the target among different blocks of trials. Search was facilitated by a word-nonword category distinction between target and distractors, particularly when target-noise feature overlap was high, but such facilitation consisted of slope reductions in an apparently serial, self-terminating search pattern as opposed to a "pop-out" effect.
\end{abstract}

In a visual search task that requires subjects to determine whether a target item is embedded in a display containing distractor items, one typically observes a performance pattern suggestive of a serial, self-terminating search. This pattern includes a linear relationship between the search time and the number of items in the display, and a 2:1 slope ratio between the trials in which the target was absent and those in which it was present. One important exception to this pattern of data has been demonstrated by experiments in which the target differs from the background items on the basis of a unique perceptual characteristic, or "feature," such as color (e.g., Treisman, 1982; Treisman \& Gelade, 1980) or closure of form (Pomerantz, 1981; Pomerantz, Sager \& Stover, 1977). In those studies, perceptually unique targets seem to "pop out" of the display without the need for an effortful serial search, thus producing either nearly flat or discernibly nonlinear relationships between search time and display size. This type of pop-out phenomenon is essentially similar to the rapid perceptual segregation of differing visual textures (Julesz, 1981).

A second apparent exception to serial visual search has been reported in experiments in which the target is a member of a different category of items from the distractor or background alternatives, such as letters embedded in digits and vice versa (Brand, 1971; Egeth, Jonides, \& Wall, 1972; Ingling, 1972; Jonides \& Gleitman, 1972, 1976). Several of those studies have reported nearly flat display size functions for targets belonging to the category different from the background items. In the case of the Jonides and Gleitman (1972) study, visual search for the

This research was supported by NSF Grants IST- 8110544 and IST8319016 to the first author. Portions of this research were presented at the 24th annual meeting of the Psychonomic Society, San Diego, California, November 1983. The authors are indebted to Julie Shleif, Doris Reed, and Kathy Fuson for assistance in data collection and analysis. Please note that Doris J. Lohr was formerly Doris Carson.

The authors' mailing addrress is: Department of Psychology, 209 Burnett Hall, University of Nebraska, Lincoln, NE 68588-0308. same target $(O)$ produced either a flat or a positive linear slope of about $24 \mathrm{msec}$ per item in the display, depending upon whether the subject had encoded it as "Oh" or "Zero" and whether the background items were digits or letters. This finding strongly suggested that the category distinction was not based upon use of a visual feature criterion which tends to distinguish letters from digits, but rather upon the ability to categorically segregate the items prior to the comparison process. Unfortunately, some more recent experiments have cast doubt on the replicability of some of these findings (Duncan, 1983; Francolini \& Egeth, 1979). These more recent studies have left open the question of whether facilitation of search resulting from a categorical distinction between the target and background items represents a true pop-out phenomenon in which serial comparisons are bypassed or merely an increase in the efficiency of conducting serial comparisons.

\section{Do Words Pop Out of Nonword Backgrounds?}

While most studies of visual search have used single characters or single element forms as targets and distractors, our present study investigated the role of word familiarity in locating familiar three-letter words in displays containing less familiar nonword trigrams. We wanted to see whether word targets would be located more efficiently than featurally similar nonword targets and, if so, whether the facilitation pattern would indicate a departure from the serial search pattern as opposed to a simple slope reduction. A true pop-out effect would be indicated by the slope of the display-size function's being nearly zero or perhaps by a discernible curvilinear function in which a decreasing slope approaches a zero asymptote.

It is important to note that, despite the recent doubts (Duncan, 1983) about whether the category uniqueness of a letter or digit target embedded in symbols of the opposite type can actually lead to a pop-out effect as some previous studies have suggested, there are additional rea- 
sons why one might expect words to be effortlessly segregated from nonwords in a visual search task. Each of the reasons is supported by some relatively widely accepted theories of human information processing, and we will consider them below.

\section{Perceptual Unitization of Words}

According to the perceptual unitization concept (LaBerge, 1981; LaBerge \& Samuels, 1974), words tend to be processed as holistic perceptual patterns as opposed to collections of individual letters. In addition to the "cognitive plausibility" of such a theory in accounting for the large increases in reading efficiency that occur with practice, experiments have shown marked changes in the focal span of attention between search tasks involving word stimuli versus nonword stimuli (LaBerge, 1983). If such unitization is what Treisman and her colleagues (e.g., Treisman \& Gelade, 1980) have termed a preattentive process, which occurs prior to the application of focal attention in serial search, it is conceivable that words functionally create their own unique perceptual features, even though they share many physical features at the level of individual letters or letter parts. The presence of the functionally created unique feature might therefore lead to a pop-out effect just as if the ink color of the target had differed from that of the background.

\section{Attentional Capture}

Attentional capture effects are well known in the auditory domain, both in experimental research and in everyday life. The presentation of one's name from an unexpected spatial location can easily interrupt attention to another conversation. The word "Help!' to a lifeguard has very fortunate, attention-capturing properties in a task that is quite analogous to a search task, since it involves a large number of potential target locations and distractors. The occurrence of capture effects in selective listening tasks (e.g., Treisman, 1964) provided one of the major initial challenges to the early selection or filter models of attention described by Broadbent (1958). In the auditory modality, there are clearly instances in which highly overlearned targets can be detected, or at least oriented toward, without prior focal attention, and such attentional shifts seem to occur involuntarily.

One set of experiments from our laboratory (Flowers, Polansky, \& Kerl, 1981) suggests the possibility of such a capture effect in the visual modality. In those experiments, which employed a partial report paradigm, subjects were required to report a string of letters cued by either a marker or tone which immediately followed a brief exposure of several letter strings. On some trials, one of the letter-string alternatives formed a familiar word. On the trials in which a familiar word occurred in a noncued location, accuracy for reporting the cued string was less than it was on the trials in which no word was present in the display. One interpretation of this "word inferiority effect" is that focal attention was involuntarily drawn by the familiar word such that subjects could not shift away to the cued position. Even a weak "capture effect" that "pulled" focal attention in a visual search task should be sufficient to improve search efficiency from that of strict serial comparisons. Short of a flat-slope "immediate pop-out," one would expect to observe a definite curvilinear display-size function if such involuntary (or perhaps voluntary) capture had occurred.

\section{The "Automatic" Encoding of Words}

According to an important theory of attention developed by Schneider and Shiffrin (1977), searching for highly overlearned visual targets which have been consistently mapped against a set of distractors does not require sequential processing. These authors have provided a large quantity of empirical data in support of this view. By analogy, words as a class of stimuli are highly overlearned, and when presented as targets in a task in which they must be discriminated from highly unfamiliar letter strings (consistently mapped) over a large number of trials, one might expect a similar display-size effect to that found in the character-detection studies of Schneider and Shiffrin (1977). With respect to word stimuli in particular, however, a relatively widely shared view among cognitive psychologists, one derived from the concept of automatic processing, is that lexical access does not require focal attention and does not necessarily require conscious identification of the word. Studies of parafoveal priming effects (e.g., Rayner, McConkie, \& Ehrlich, 1978) emphasize the former point, whereas some of the highly controversial "subliminal" priming experiments (e.g., Marcel, 1983) make a somewhat less universally accepted case for the latter. In a visual search task, in which the target is the only word stimulus that could occur in a display, positive detection does not logically require the target's identification or even its localization; simply noting that lexical access has occurred would be sufficient. Thus, if lexical access can occur rapidly, and independently of spatial attention, and if that information could be used to generate a motor response, then word targets embedded in nonword distractors ought not to create a data pattern suggesting serial self-terminating search, but something much closer to a pop-out effect.

In summary, there is a relatively compelling body of cognitive theory which suggests that locating words among nonword targets need not require a serial search, irrespective of challenges to the concept of perceptual selectivity between letters and digits. It should be noted, however, that the concepts of perceptual unitization, attentional capture and "automatic" encoding are not mutually exclusive theoretical categories. For instance, both perceptual unitization and attentional capture imply the existence of some form of lexical analysis prior to focal attentioni.e., "automatic" encoding. However, the question of exactly how preattentive analysis of a word leads to a popout effect is not relevant unless the empirical effect can be demonstrated. Thus, the purpose of the present study 
was to see whether a word-nonword distinction would lead to a pop-out effect and, if so, to delineate the boundary conditions within which it could be obtained.

\section{EXPERIMENT 1}

The purpose of our first experiment was to assess the functional relationship between display size and search time for word and nonword targets embedded in word or nonword distractors. We elected to treat this experiment as essentially a psychophysical measurement problem, employing a limited number of highly trained subjects and a relatively large number of trials, in order to provide stable functions for individual subjects. While we were primarily interested in the possible pop-out effects of a word-nonword distinction between target and background items, we were also interested in comparing the magnitude of the category effects with those attributable to different levels of physical feature confusability between the target and background. By deliberately manipulating the physical feature overlap between the target and background items, we sought to create different levels of "room for improvement" with the category distinction. It seemed possible that evidence for a departure from a sequential search process might be difficult to detect in a situation in which the search rate through the background items was very rapid.

\section{Method}

Subjects. Two female graduate students and one male faculty member each served in a total of 13 sessions (including an initial practice session used to familiarize them with the task). Each session lasted about $1.5 \mathrm{~h}$. Each of the three subjects contributed a total of 5,760 reaction times during the course of the experiment. All subjects had normal or corrected vision (one wore corrective lenses during the study), and English as their native language.

Task and Apparatus. Stimuli were displayed on a Zenith Model ZVM-121 video monitor (P31 phosphor) in normal uppercase characters generated by an Apple II Plus computer. Each stimulus display consisted of $1,2,3,4,6$, or 12 trigrams, equally spaced around a nearly circular "clockface" pattern around the center of the video screen. Although precise head positioning was not controlled, the maximum visual angle between most distant letters in a display was approximately $9.5^{\circ}$ for a typical subject.

The onset of each stimulus display was preceded by a warning tone from the computer approximately $1 \mathrm{sec}$ prior to display onset. At the onset of the display, the subjects searched for the presence of a prespecified target trigram and pressed one of two keys on the computer keyboard to indicate presence or absence of the target. The computer measured the reaction time (RT) from display onset to the keypress, to the nearest millisecond, by means of a California Computer Systems Model 7440 timer.

On trials on which the subject's response was correct, the RT was displayed to the subject for approximately $1.5 \mathrm{sec}$ following the trial. On trials on which an incorrect response was made, the computer emitted a warning tone and a flashing "OOPS!" message was displayed for about $2.5 \mathrm{sec}$. Following either the RT feedback or the error admonition message, the video screen remained blank for approximately $3 \mathrm{sec}$ before the warning tone signaled the onset of the next trial. Trials on which errors were reinserted randomly into the trial sequence, and thus only RT from correct trials were used in the primary data analysis. However, the computer did count the number of errors that occurred for each combination of independent variables, and those data were printed out following each experimental session.

Independent variables. The subjects searched for six different trigram targets during the course of the experiment. These targets were the words SEX, BOY, AND YES, and the nonwords SFX, BDY, AND YLS. Thus, the target category (word vs. nonword) was one of the independent variables. The subjects searched for a single target for two experimental sessions ( 960 trials) before changing to a new target. The order of targets assigned to each subject was constrained such that it alternated between word and nonword targets, and no physically similar word-nonword pairs (e.g., SEX, SFX) were searched for on adjacent trial blocks.

The second independent variable was the background. For each of the 12 target alternatives, four different sets of background items served as the noise or distractor items. These sets of background items were held constant within blocks of trials, and the subject was allowed to view the background items before beginning a block of trials. Each of these distractor sets is listed in Appendix A. These four different sets of distractors for each target constituted four different levels of physical similarity between the target trigram and distractors. For one level, the high-similarity nonwords, the distractor items contained the same letters as the target, but in a different order. Thus, the high-similarity-nonword distractors constituted a very high degree of physical feature overlap with the target item. Analysis of spatial arrangement (thought by Treisman and others to absolutely require postattentive scrutiny) was necessary before a distractor item could be rejected as a nontarget. For a second level of target-background similarity, the medium-similaritynonword background, each alternative distractor item shared one letter in common with the target, while the remaining two letters of each distractor were moderately dissimilar to the letters contained in the target. For the low-similarity-nonword distractors, all letters that made up the distractor trigrams were moderately dissimilar to the letters of the target. For the final background condition, the low-similarity words, we selected trigrams that were moderately high- to high-frequency English words, which were composed of letters of at least moderate dissimilarity to those contained in the target. Distractor items used with each target alternative and background condition are listed in Appendix $\mathrm{A}$. We presumed that the first three conditions would produce large differences in search time owing to simple visual feature confusability differences, and hence widely different amounts of "room for improvement," with any familiarity effect arising from the search for a word as opposed to a nonsense trigram. Additionally, we were interested in whether the word background condition might eliminate or reverse any familiarity effects observed in the other background conditions.

The two remaining independent variables, display size and target presence varied within trial blocks. Display size (number of trigrams in the display) was $1,2,3,4,6$, or 12 , with each level occurring five times during the 60 experimental trials within a block for target-present and for target-absent trials. The three extra warmup trials that occurred at the beginning of each trial block (and were not analyzed) were selected randomly from the 60 experimental triais. The order to the stimulus displays in the trial blocks was determined by a pseudorandomly generated shuffling program.

\section{Results and Discussion}

For each of the three subjects, mean RTs were computed for each combination of display size, target presence, and background type, separately for each of the six target alternatives. For each of the three subjects, there were essentially no differences in performance attributable to which of the three word targets they were searching for, and the same held true for the nonword targets. Moreover, although there were some slight differences among the three subjects in overall level of performance, 
the qualitative pattern of effects was essentially identical for the three subjects. Except where noted, each of the main effects and interactions described in this experiment were statistically significant $(p<.01)$ on the basis of individual within-subject analyses, as well as the group comparisons reported in this text. Thus, to simplify data presentation, we will present means for the three-subject group collapsed across the three word targets and the three nonword targets.

Figures 1-4 plot the mean RT as a function of display size, target presence, and background, illustrating the differences between word and nonword targets within each background condition. Table 1 presents the mean slopes and intercepts of the least squares regression equations (computed for individual subjects) relating RT and display size. Inspection of these tables and figures reveals several robust characteristics of the data which were consistent across individual subjects and stimuli. These include a highly linear relationship between display size and RT, and a steeper slope for target-absent than for targetpresent trials. This pattern occurred for each subject and every individual target stimulus. Four analyses of variance (one for each background type) each revealed highly

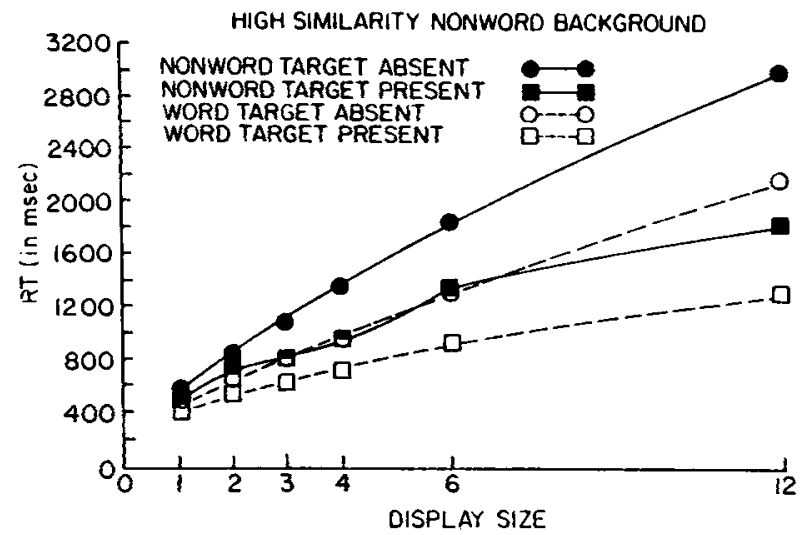

Figure 1. Mean RT plotted as a function of display size for the high-similarity-nonword background condition in Experiment 1.

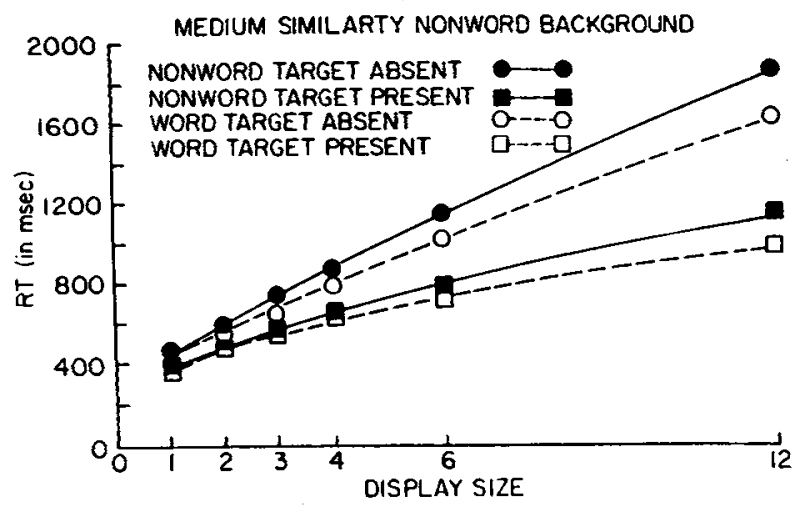

Figure 2. Mean RT plotted as a function of display size for the medium-similarity-nonword background in Experiment 1.

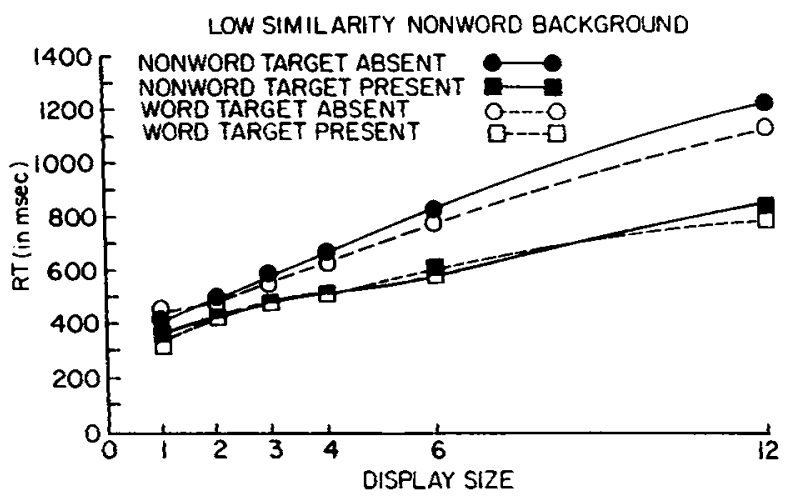

Figure 3. Mean RT plotted as a function of display size for the dissimilar-nonword background in Experiment 1.

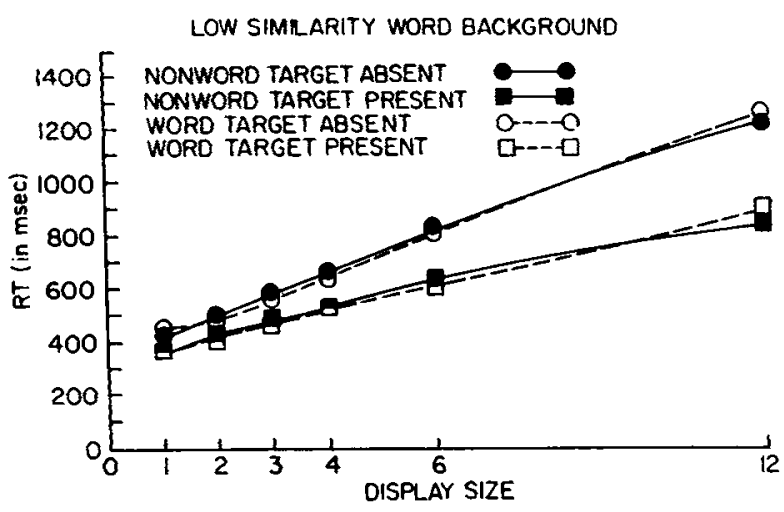

Figure 4. Mean RT plotted as a function of display size for the word background condition of Experiment 1 .

significant effects of display size, target presence, and their interaction $(p<.001$ for the interaction in each case). Table 1 shows that slope ratios between targetabsent and target-present trials were very close to the 2:1 ratio for the high-similarity-nonword and medium-similarity-nonword background conditions, although slightly less for the low-similarity-nonword and low-similarityword backgrounds (but in no case less than 1.5:1). Such a pattern is quite consistent with the predictions of the serial self-terminating search model, and there is little evidence for a "pop-out" effect with any combination of target and background.

The finding of greatest interest from Experiment 1, however, was the substantial difference in slopes between word and nonword targets, which occurred most notably with the high-similarity-nonword and medium-similaritynonword backgrounds. For these two background conditions, analysis of variance showed highly significant display size $\times$ target category interactions $[F(5,10)=38.46$ and $15.41, p<.001$ in each case]. It is clear, however, from the visual presentation of these data in Figures 1 and 2 , and from the correlation coefficients listed in Table 1 , that this substantial slope reduction, which occurred when subjects searched for a word target as opposed to a non- 
Table 1

Mean Slopes and Intercepts (in Milliseconds) and Correlation Coefficients Relating Search Time to Display Size for Each Combination of Background and Target Category in Experiment 1

\begin{tabular}{lrrrrrrr} 
& \multicolumn{3}{c}{ Word Target } & & \multicolumn{3}{c}{ Nonword Target } \\
\cline { 2 - 5 } \cline { 5 - 7 } Background & Slope & Intercept & $\mathrm{r}$ & Slope & Intercept & $\mathrm{r}$ \\
\cline { 2 - 7 } Hi Sim Nw & 80 & 380 & .93 & 112 & 483 & .89 \\
Med Sim Nw & 52 & 372 & .84 & 67 & 357 & .90 \\
Lo Sim Nw & 41 & 338 & .89 & 43 & 341 & .88 \\
Lo Sim Wd & 47 & 329 & .87 & 42 & 357 & .86 \\
& & & Target & Absent & & \\
Hi Sim Nw & 149 & 369 & .98 & 218 & 425 & .98 \\
Med Sim Nw & 106 & 358 & .97 & 126 & 356 & .98 \\
Lo Sim Nw & 63 & 378 & .94 & 71 & 369 & .95 \\
Lo Sim Wd & 75 & 345 & .96 & 71 & 368 & .96 \\
\hline
\end{tabular}

word containing highly similar visual features, did not produce any departures from the pattern of an essentially linear relationship between search time and display size, or a departure from the $2: 1$ slope ratios between targetabsent and target-present trials. It would thus appear that the most parsimonious explanation for the substantial familiarity effect is an increase in the speed with which serial comparisons can be made, as opposed to a departure from the serial search process itself.

For the low-similarity-nonword background condition, which led to considerably faster search times overall, the display size $\times$ target category interaction was substantially attenuated, occurring for only two of the three subjects, and the overall statistical test of the interaction among the three subjects was not significant $[F(5,10)=$ 1.72]. When the background items were words themselves, there was a suggestion of a reversal of the target familiarity effect; the slopes were slightly steeper for word than for nonword targets, although this effect was not statistically significant $[F(5,10)=1.69]$.

With the possible exception of the high-similarity-nonword background, the intercept values listed in Table 1 show that the target category had relatively little effect on intercepts; no statistically significant differences in intercept values between word and nonword targets were noted. The apparent difference in intercepts for word and nonword targets with the high-similarity-nonword background was primarily attributable to large differences produced by only one of the three subjects. ANOVA comparisons of intercepts among the three subjects for the high-similarity background does not show this effect to be significant $[F(1,2)=3.3]$, although the lack of degrees of freedom does not provide a very powerful test. If this intercept effect reflects a real phenomenon, it is puzzling why a similar trend would not occur for the mediumsimilarity-nonword background, which obviously it did not.

Apparently, the advantage for searching for a word target is most pronounced when high levels of visual confusability between the target and the background lead to a large amount of room for improvement. Nevertheless, of potential interest is the trend suggesting a reversal of the word-nonword target effect when targets are being searched for among background items that are themselves words. One possible "nonperceptual" interpretation of the faster search times for word targets is that the lesser memory-load requirements of the word target (one vs. three "chunks") leads to a general increase in resources which can be allocated to other components of the search task. Finding that nonword targets lead to faster search when the distractor items are familiar words would contradict such a memory-load interpretation of the familiarity effect, and tend to favor a model based upon an increase in the efficiency of testing the display items.

The design of Experiment 1 was not ideally suited to making such a test, since no attempt was made to control precisely the visual features of the distractor items between the low-similarity-nonword and low-similarityword background conditions. Furthermore, a three-subject sample is not appropriate for making a conclusive parametric test. Thus, a comparison of search times for word and nonword targets in word and nonword backgrounds was the central focus of Experiment 2.

Error rates in Experiment 1 averaged $8.1 \%$ for the highsimilarity-nonword background, $7.4 \%$ for the mediumsimilarity-nonword background, $5.1 \%$ for the low-similarity-nonword background, and $6.3 \%$ for the lowsimilarity-word background. Although these rates are too low for meaningful comparisons of error frequencies for word and nonword targets to be made, it should be noted that errors were not equally distributed across display size levels in target-absent vs. target-present trials. Specifically, subjects tended to make more errors on large-display-size trials when the target was present, but made the greatest proportion of errors for display size 1 on the target-absent trials, as shown in Figure 5-8. Informal comments from subjects suggested a tendency to shift response expectancies as a function of time spent searching, which would account for the imbalance in error distributions across display-size levels. One might expect the occurrence of such criterion shifts or response expectancy effects to result in differential speed-accuracy tradeoffs

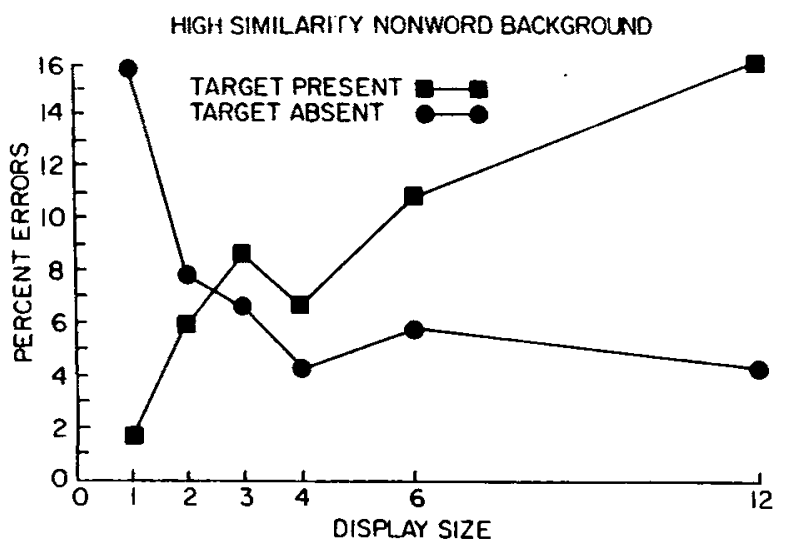

Figure 5. Percentage errors plotted as a function of display size for the anagram background condition of Experiment 1. 


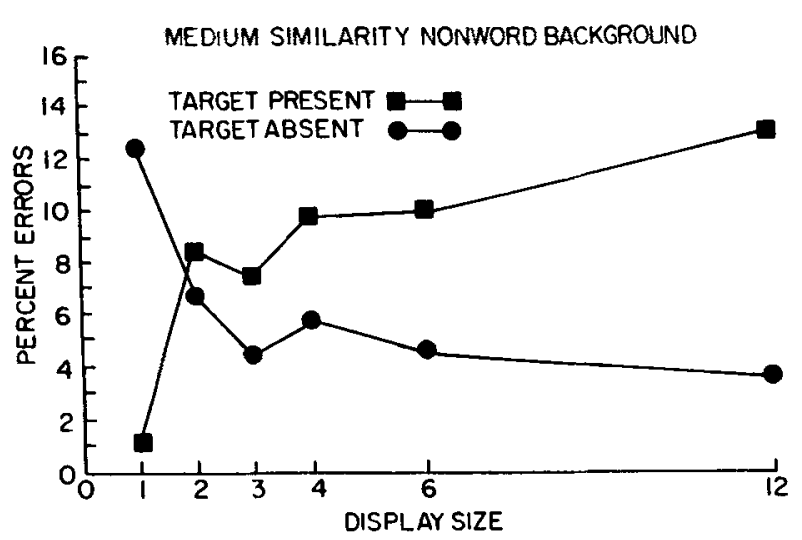

Figure 6. Percentage errors plotted as a function of display size for the medium-similarity-nonword background condition of Experiment 1.

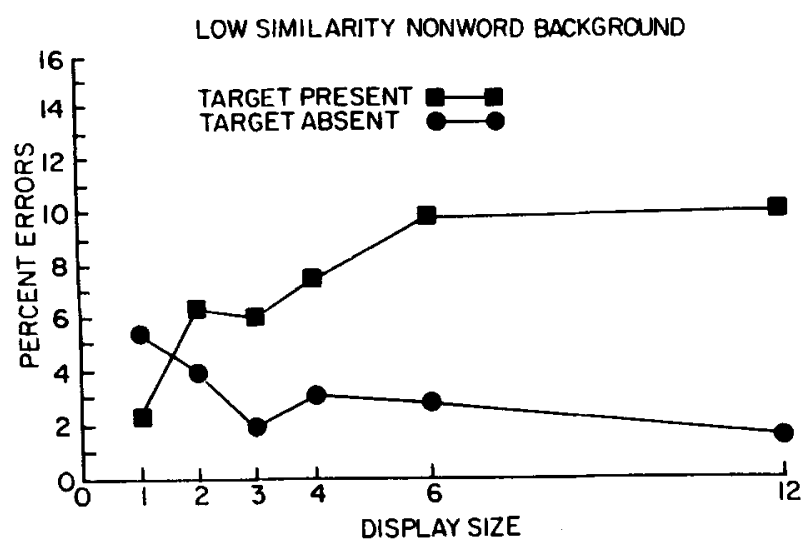

Figure 7. Percentage errors plotted as a function of display size for the low-similarity-nonword background condition of Experiment 1.

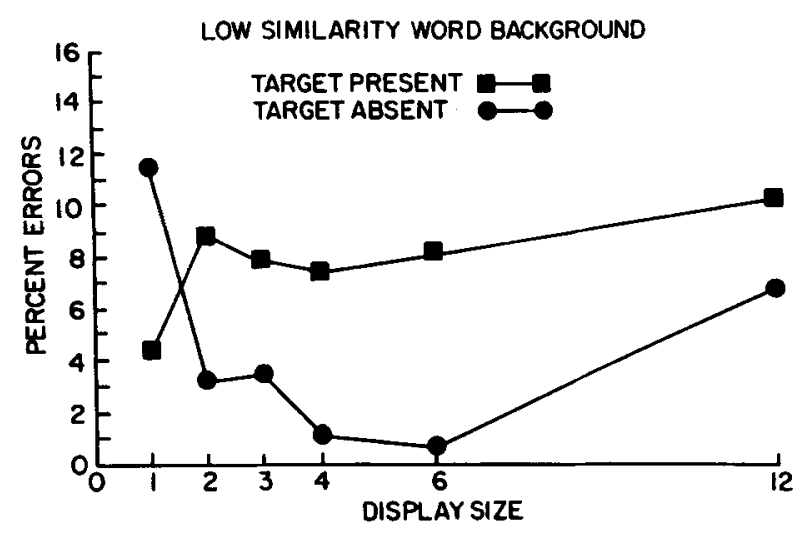

Figure 8. Percentage errors plotted as a function of display size for the low-similarity-word background condition of Experiment 1.

across display-size levels, which might produce a curvilinear relationship between display size and RT even if the search process is essentially serial in nature. The fact that our data illustrate a strong linear relationship between display size and RT and a close to $2: 1$ ratio of target-absent to target-present slope values, despite the possible existence of such tradeoffs, argues quite strongly against the existence of a pop-out effect which bypasses the search process.

\section{EXPERIMENT 2}

The results from Experiment 1 suggested that the advantage of searching for a word target as opposed to a nonword containing nearly identical visual features was lost, or perhaps even reversed, when the distractor items were themselves words. Experiment 2 was conducted to provide a more powerful test of this trend, using a larger number of subjects, who searched word and nonword distractor sets that contained identical letters (i.e., the nonwords were anagrams of the word distractors).

\section{Method}

Subjects. Six undergraduate and graduate student volunteers each served in six experimental sessions lasting about $1 \mathrm{~h}$ each. They were each paid $\$ 16.50$ for their services. All subjects had normal or corrected vision and English as their native language.

Task and Apparatus. The task and method of stimulus presentation were identical to that of Experiment 1 . The only differences in general procedure between Experiments 1 and 2 were the target and distractor items, which are described below.

Independent variables. The independent variables used in Experiment 2 were target category (word or nonword), background category (words or nonwords), target presence, and display size. The target items used in Experiment 2 included the three words SEX, DOG, and THE, and the nonwords SFX, DCG, and THL. As in Experiment 1, the subjects completed all trials for a single search target before shifting to a new target item, and pairs of similar words and nonwords were not presented in contiguous sessions.

For each target alternative, two sets of distractor items were used. These included moderately high-frequency English three-letter words, containing letters different from those included in the target, and nonsense trigrams that were anagrams of the words used for the word distractors. The actual distractor sets used with each target are presented in Appendix B.

As in Experiment 1, target-present and target-absent trials each occurred five times paired with each of the six levels of display size $(1,2,3,4,6$, or 12 items), within each 60 -trial block. As in Experiment 1, three additional warm-up trials were included at the beginning of each trial block, but were not included in analysis.

\section{Results and Discussion}

Figures 9-11 plot the mean RT as a function of display size, background, and target presence. Separate plots are shown for the word targets and the nonword targets. Table 2 presents the mean slopes, intercepts, and Pearson correlation coefficients for least squares linear regressions for the mean RTs averaged across stimuli and subjects. It can be seen that the functions relating RT to display size are highly linear, with slope ratios ranging from 1.9:1 to 2.2:1 between target-absent and target-present trials-a pattern similar to that of Experiment 1.' Error rates were somewhat less than those found in Experiment 1-7.4\% averaged across all levels of display size and targetbackground combination. As in Experiment 1, there was a tendency for the errors to increase with display size for 


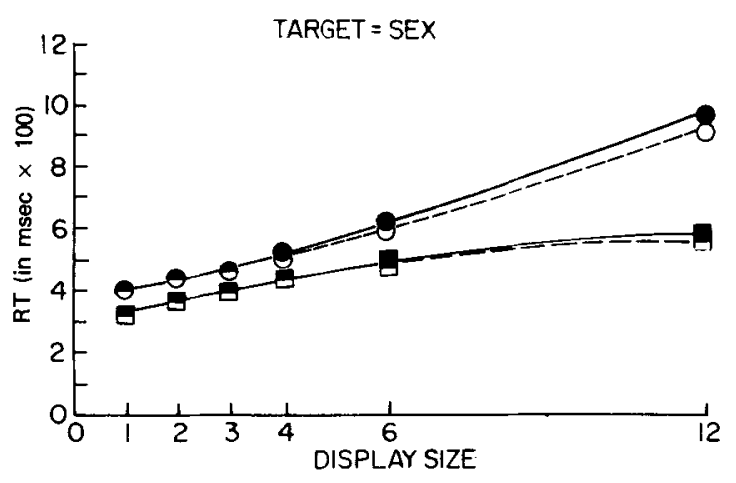

target-present trials (1.2\% for display size 1 to $10.4 \%$ for display size 12), whereas an opposite pattern held for target-absent trials $(9.8 \%$ to $2.4 \%)$.

Most importantly, however, the RT data illustrate a significant interaction between target category (word vs. nonword), background (word vs. nonword), and display size $[F(5,25)=12.97, p<.01]$. Furthermore, analysis of slopes computed individually for each subject also showed a significant interaction between target category and background $[F(1,5)=27.17, p<.01]$. Searching for word targets among word distractors or nonword targets among nonword distractors produced steeper display-size functions than did searching for targets that differed in the conceptual category from the background items. Inspection of Figures 5-8 shows that this relationship held for all three target pairs. However, it is also apparent from these figures that the effect is essentially concentrated in the target-absent trials. Analysis of variance of the RTs did reveal a significant four-way interaction between target category, background, display size, and target presence. Analysis of variance of the individually computed slopes revealed a significant target $\times$ background $\times$ target presence interaction as well $[F(1,5)=11.37, p<.05]$. Thus, although the relationship between target category and category of the distractor items affected search time to some extent regardless of whether the target was a word or a nonword, this conceptual category effect contributed

Figure 9. Mean RT plotted as a function of display size, background, and target presence for the target stimuli SEX and SFX in Experiment 2.

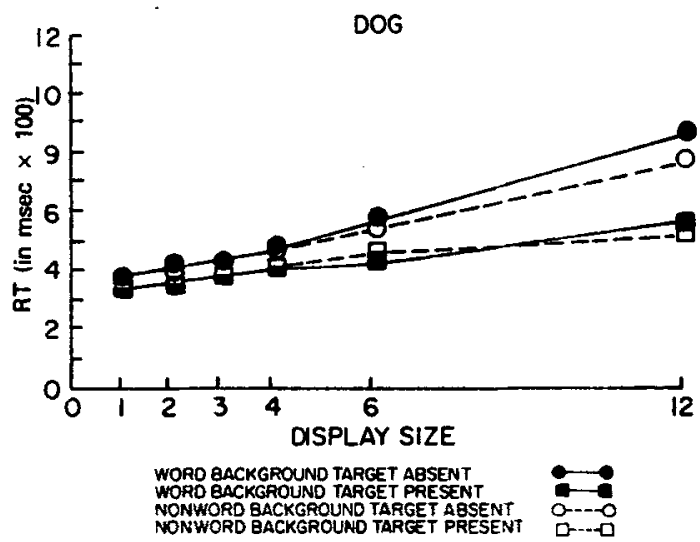

much less to performance than it did in Experiment 1 for
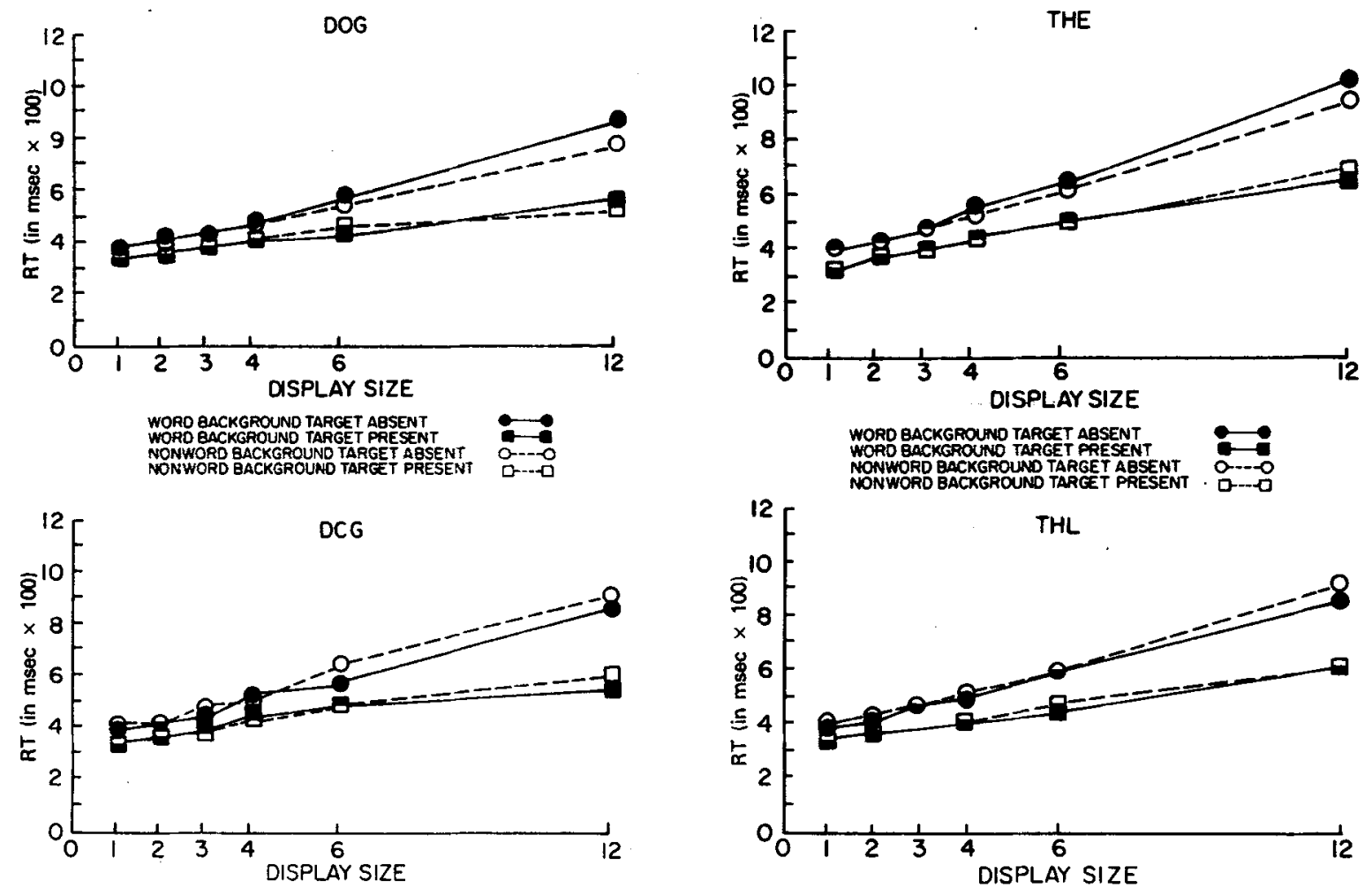

Figure 10. Mean RT for the targets DOG and DCG in Experiment 2.

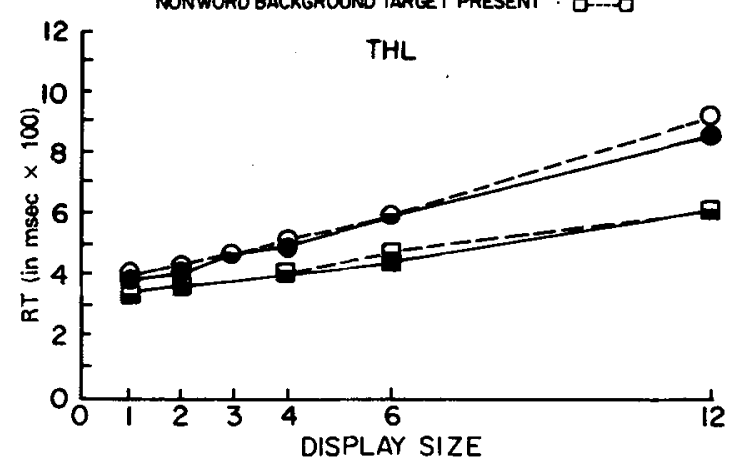

Figure 11. Mean RT for the targets THE and THL in Experiment 2. 
Table 2

Mean Slopes and Intercepts (in Milliseconds) and Correlation Coefficients Relating Search Time to Display Size for Each Combination of Target Category and Background Condition in Experiment 2

\begin{tabular}{lccccccc}
\hline & \multicolumn{3}{c}{ Target Present } & & \multicolumn{3}{c}{ Target Absent } \\
\cline { 2 - 4 } Condition & Slope & Intercept & $\mathrm{r}$ & & Slope & Intercept & $\mathrm{r}$ \\
\hline WT-WB & 24.3 & 315.7 & .90 & 51.9 & 315.8 & .95 \\
WT-NB & 24.1 & 319.3 & .90 & 45.0 & 329.6 & .95 \\
NT-WB & 22.9 & 326.9 & .90 & 46.5 & 330.5 & .95 \\
NT-NB & 24.0 & 323.1 & .93 & 51.6 & 323.8 & .95 \\
\hline
\end{tabular}

the conditions in which a substantial degree of visual confusability existed between target and background.

\section{EXPERIMENT 3}

Experiment 3 was conducted to provide further substantiation of the interaction between the target category (word vs. nonword) and the category of the background items, by selecting targets and background items from the same set of three letters. Presumably the use of such stimulus displays would lead to levels of task difficulty more like those of the high-similarity-nonword and medium-similarity-nonword conditions of Experiment 1, providing greater room for improvement through use of a category distinction between target and distractor items.

\section{Method}

Subjects. Twelve University students served in two experimental sessions lasting about $2.0 \mathrm{~h}$ each, and were paid $\$ 14$ for their services. All subjects had normal or corrected vision and English as their native language.

Task and Experimental design. Experiment 3 used the same general procedure and apparatus, and manipulated the same independent variables, as Experiment 2; the only differences were in the target and background items used and in their assignment to subjects during the two sessions. The target and background items were selected from the six permutations of the letters $R, A$, and $T$. It may be noted that these permutations include the three words RAT, ART, and TAR, plus the three nonwords RTA, TRA, and ATR. Four subjects searched for the targets RAT and RTA (in separate sessions, with the order counterbalanced), four subjects searched for TAR and TRA, and the remaining four subjects searched for ART and ATR. For half the trial blocks within each session, the distractor items were the two nonword anagrams of the target that did not share the common first letter (e.g., the strings TRA and ATR for the targets RAT or RTA); for the remaining trial blocks, the distractor items were the two word anagrams of the target that did not share a common first letter (e.g., TAR and ART with the targets RAT or RTA).

Each of the experimental sessions, during which the subject searched for a single target, included 10 blocks of 63 trials (for which the first three practice trials were discarded). These included five word-background and five nonword-background blocks, presented in alternation. The first two blocks in each session (one word background and one nonword background) were considered practice trials and excluded from analysis. As in Experiments 1 and 2 , the 60 trials within each block that were included for data consisted of five replications of the 12 different combinations of display size and target presence. Error trials were reinserted randomly, as in the two previous experiments.

\section{Results and Discussion}

Figure 12 plots the mean RTs, averaged across subjects for each combination of background type and target presence, as a function of display size. The top and bottom panels illustrate data obtained with word and nonword targets, respectively. It can be seen that an interaction between target category and background category, similar to that of Experiment 2, occurred. When the search was for word targets, the slopes produced were steeper when the distractors were words than when they were nonwords; the situation was reversed when the targets were nonwords. There was a significant three-way interaction between target category, background category, and display size $[F(5,45)=10.3 ; p<.01]$, but no significant four-way interaction with subject group (i.e., which of the three target pairs a subject was assigned to). Table 3 presents the mean slopes, intercepts, and values of Pearson $r$ based on computations from individual subjects' data, for each combination of word and background category. It can be seen that the slopes are considerably larger than those obtained with the stimuli used in Experiment 2, owing to the much greater feature overlap between targets and distractors. ${ }^{2}$ Even with this greater "room for improvement," the slope differences caused by the category distinctions for the target-present trials were quite small, relative to the target-absent trials. Nevertheless,
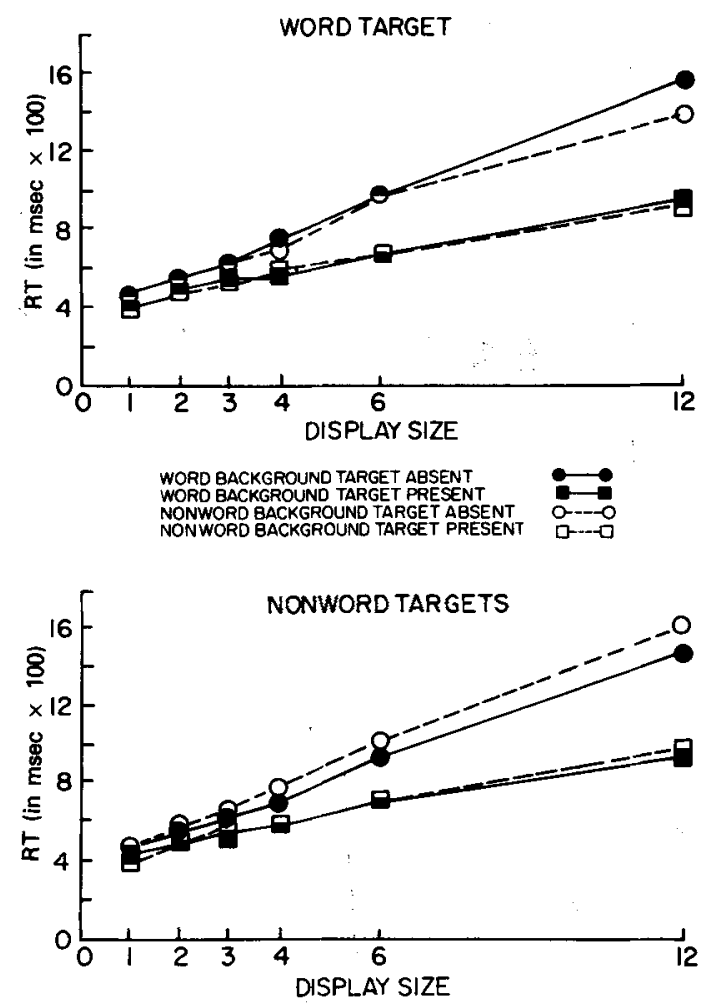

Figure 12. Mean RT for the word and nonword targets plotted as a function of display size, background, and target presence in Experiment 3. 
Table 3

Mean Slopes and Intercepts (in Milliseconds) and Correlation Coefficients Relating Search Time to Display Size for Each Combination of Target Category and Background Condition In Experiment 3

\begin{tabular}{lcccrrrr} 
& \multicolumn{3}{c}{ Target Present } & & \multicolumn{3}{c}{ Target Absent } \\
\cline { 2 - 4 } \cline { 6 - 8 } Condition & Slope & Intercept & $\mathrm{r}$ & & Slope & Intercept & $\mathrm{r}$ \\
\hline WT-WB & 49.6 & 371.3 & .87 & & 102.1 & 346.7 & .97 \\
WT-NB & 48.4 & 366.8 & .89 & 87.1 & 379.8 & .95 \\
NT-WB & 48.2 & 389.6 & .87 & 91.6 & 364.9 & .97 \\
NT-NB & 50.9 & 353.7 & .87 & 100.4 & 370.9 & .96 \\
\hline
\end{tabular}

the strong linear component of the display-size function, taken together with the lack of discernible nonlinear trends and close to 2:1 ratios in target-absent to target-present slopes point to the continuance of a serial, self-terminating pattern of search in which the word-nonword category distinction serves primarily to expedite the serial comparisons.

Error rates followed the same general pattern of Experiments 1 and 2 . The overall error rate was $10.0 \%$ and ranged from $1.8 \%$ to $16.4 \%$ across display-size levels for target-present trials, and from $15.4 \%$ to $4.8 \%$ in targetabsent trials.

\section{GENERAL DISCUSSION}

Our original objective in conducting these experiments was to see whether a familiar-word target could be detected within an array of unfamiliar letter strings without using the typical, presumably serial, search process associated with locating visual targets among background elements that share similar features. In this regard, our data do not suggest that any sort of "pop-out" effect, similar to that associated with detecting certain types of forms or textures that have uniqui features (Julesz, 1981; Treisman \& Gelade, 1980), occurred when the target could be distinguished from the background items on the basis of a word-nonword distinction. Regardless of the relationship between the target and background, the functions relating display size to RT were highly linear, and exhibited the 2:1 slope ratio between target-absent and target-present trials predicted by a serial self-terminating search process.

However, we did find a rather dramatic effect of the category of the target (word vs. nonword) on the speed with which the apparently serial comparisons could be made. In Experiment 1, the simple deletion of a single feature of a single letter of a three-letter target trigram, (SEX vs. SFX) or the simple perturbation of the features of a single letter (BOY vs. BDY) caused nearly a $50 \%$ reduction in the per item search time, when the distractors were anagrams of the target, and about a $20 \%$ reduction when the background items each shared a single letter in common with the target. Given the very minor physical change that transformed the target from a word into a nonword, it is very clear that some aspect of the lexical-nonlexical category distinction is crucially affect- ing the decision process by which distractor items are rejected.

Our resuits bear a resemblance to the findings of an experiment by Karlin and Bower (1976). In Karlin and Bower's study, subjects searched for a target set of words which either differed from the distractor items in terms of a semantic category distinction or belonged to the same category as the distractors. Karlin and Bower found that when subjects were required to search for a single word target belonging to a semantic category different from that of distractor words in the background (e.g., looking for the word "spruce" among color-name distractors, display-size functions were essentially identical to those obtained when the category of the target and the distractors was identical. When performance was degraded by an increase in target set size to three or five items, however, substantial slope reductions occurred when the target belonged to a category different from that of the distractors, although the functions relating RT to display size were still highly linear, with a 2:1 slope ratio between target-present and target-absent trials.

To interpret their findings, Karlin and Bower suggested that the comparison between display items and memory involves two qualitatively different types of tests, a categorization test and a perceptual feature test, which are made in parallel. Search proceeds to the next item in the display whenever either of the processes makes a negative decision, and terminates whenever either process produces a "yes" decision. Under conditions which lead to a slow perceptual match, the categorization test is likely to finish first; hence, target and background conditions for which the categorization process can be used as a valid means of separating targets and nontargets will lead to shallower RT increments as a function of display size. When the category distinction is not a valid cue, subjects must always use the slower, perceptual match process. Apparently, given the perceptual distinctiveness of the target and distractor items used in Karlin and Bower's study, time advantage of the category comparison became useful only when the "physical" matching strategy was slowed by an increased memory load. In our experiments (which used a target set size of one item exclusively), it appears that the use of word/nonword category distinction became increasingly useful as the per item search time was slowed by increasing the physical feature overlap between target and distractor.

If a dual-process "race" model is an appropriate representation of the mental activity associated with our task, then it seems that at least some form of lexical categorization processes can proceed independently of the feature comparisons necessary for physically matching a nonword string. Such a process need not, however, imply the type of preattentive pop-out effect found in texture-discrimination tasks and related forms of effortless visual search. We would thus argue that category effects and extended practice effects in search tasks (with consistently mapped stimuli) probably do not totally remove the need for allocating spatial attention. Category effects in visual search 
that are based upon learned relationships and extensive practice appear to us to be a class of psychological phenomena that are fundamentally different from that of the pop-out effects or the texture segregation resulting from the detection of unique color characteristics or certain biologically relevant properties of visual form, such as closure.

\section{REFERENCES}

BraND, J. (1971). Categorization without identification in visual search. Quarterly Joumal of Experimental Psychology, 23, 178-186.

Broadbent, D. E. (1958). Perception and communication. New York: Pergamon Press.

DUNCAN, J. (1983). Category effects in visual search: A failure to replicate the "oh-zero" phenomenon. Perception \& Psychophysics, 34, 221-232.

EGeTH, H., Jonides, J., \& WALL, S. (1972). Parallel processing of multielement displays. Cognitive Psychology, 3, 674-698.

Flowers, J. H., Polansky, M. L., \& Kerl, S. (1981). Familiarity, redundancy, and the spatial control of visual attention. Journal of Experimental Psychology: Human Perception and Performance, 7, 157-166.

Francolini, C., \& Egeth, H. (1979). Perceptual selectivity is task dependent: The pop-out effect poops out. Perception \& Psychophysics, 25, 99-110.

INGLING, N. W. (1972). Categorization: A mechanism for rapid information processing. Journal of Experimental Psychology, 94, 239-243.

JoniDES, J., \& GleitMAN, H. (1972). A conceptual category effect in visual search: $O$ as letter or as digit. Perception \& Psychophysics, $12,457-460$.

Jonides, J., \& Gleitman, H. (1976). The benefit of categorization in visual search. Perception \& Psychophysics, 20, 289-298.

JuLEsz, B. (1981). Textons, the elements of texture perception and their interactions. Nature, 290, 91-97.

KARLIN, M., \& BoWER, G. (1976). Semantic category effects in visual word search. Perception \& Psychophysics, 19, 417-424.

LABERGE, D. (1981). Unitization and automaticity in perception. In J. H. Flowers (Ed.), 1980 Nebraska Symposium on Motivation: Cognitive Processes (Vol. 28, pp. 53-71). Lincoln: University of Nebraska Press.

LABERGE, D. (1983). Spatial extent of attention to letters and words.
Journal of Experimental Psychology: Human Perception \& Performance, 9, 371-379.

LABERGE, D., \& SAMUELs, J. J. (1974). Toward a theory of automatic information processing in reading. Cognitive Psychology, 6, 293-323.

MARCEL, A. J. (1983). Conscious and unconscious perception: Experiments on visual masking and word recognition. Cognitive Psychotogy, 15, 197-237.

Pomerantz, J. R. (1981). Perceptual organization in information processing. In M. Kubovy \& J. Pomerantz, J. (Eds.), Perceptual organization. Hillsdale, NJ: Erlbaum.

Pomerantz, J. R., Sager, L. C., \& Stover, R, J. (1977). Perception of wholes and their component parts: Some configural superiority effects. Joumal of Experimental Psychology: Human Perception \& Performance, 3, 422-435.

Rayner, K., McConkie, G. W., \& Ehrlich, S. (1978). Eye movements and integrating information across fixations. Journal of Experimental Psychology: Human Perception \& Performance, 4, 529-544.

SCHNeIDER, W., \& ShIfFrun, R. W. (1977). Controlled and automatic human information processing: I. Decision, search, and attention. Psychological Review, 84, 1-66.

Treisman, A. M. (1964). Verbal cues, language and meaning in selective attention. American Journal of Psychology, 77, 206-219.

Treisman, A. M. (1982). Perceptual grouping and attention in visual search for features and objects. Journal of Experimental Psychology: Human Perception \& Performance, 8, 194-214.

Treisman, A. M., Gelade, G. (1980). A feature integration theory of attention. Cognitive Psychology, 12, 97-136.

\section{NOTES}

1. There were no significant differences among intercept values attributable to either target or background in Experiment 2 .

2. As in Experiment 2, intercept differences were nonsignificant. Given that the target background similarity is comparable to that of the highsimilarity nonword condition of Experiment 1, the trend toward an elevated intercept observed with the nonword targets in Experiment 1 does not appear to be a generalized effect.

(Manuscript received September 3, 1984; revision accepted for publication May 16, 1985.) 
APPENDIX A

Stimulus Materials Used in Experiment 1

\begin{tabular}{|c|c|c|c|c|c|c|c|c|c|c|c|c|}
\hline Target & \multicolumn{12}{|c|}{ Distractors } \\
\hline \multicolumn{13}{|c|}{ High-Similarity-Nonword Background } \\
\hline $\begin{array}{l}\text { SEX } \\
\text { SFX } \\
\text { BOY } \\
\text { BDY } \\
\text { YES } \\
\text { YLS }\end{array}$ & $\begin{array}{l}\text { SXE, } \\
\text { SFX, } \\
\text { BYO, } \\
\text { BYD, } \\
\text { YSE, } \\
\text { YSL, }\end{array}$ & $\begin{array}{l}\text { EXS, } \\
\text { FXS, } \\
\text { OBY, } \\
\text { DBY, } \\
\text { SEY, } \\
\text { SLY, }\end{array}$ & $\begin{array}{l}\text { EXS, } \\
\text { FSX, } \\
\text { OYB, } \\
\text { DYB, } \\
\text { SYE, } \\
\text { SYL, }\end{array}$ & $\begin{array}{l}\text { XES, } \\
\text { XFS, } \\
\text { YOB, } \\
\text { YDB, } \\
\text { EYS, } \\
\text { LYS, }\end{array}$ & $\begin{array}{l}\text { XSE } \\
\text { XSF } \\
\text { YBO } \\
\text { YBD } \\
\text { ESY } \\
\text { LSY }\end{array}$ & & & & & & & \\
\hline \multicolumn{13}{|c|}{ Medium-Similarity-Nonword Background } \\
\hline $\begin{array}{l}\text { SEX } \\
\text { SFX } \\
\text { BOY } \\
\text { BDY } \\
\text { YES } \\
\text { YLS }\end{array}$ & $\begin{array}{l}\text { SNR, } \\
\text { SNR, } \\
\text { BLQ, } \\
\text { BLQ, } \\
\text { YWU, } \\
\text { YWU, }\end{array}$ & $\begin{array}{l}\text { SUQ, } \\
\text { SUQ, } \\
\text { BKR, } \\
\text { BKR, } \\
\text { WMV, } \\
\text { YMV, }\end{array}$ & $\begin{array}{l}\text { MEP, } \\
\text { MFP, } \\
\text { VOS, } \\
\text { VDS, } \\
\text { CEI, } \\
\text { CLI, }\end{array}$ & $\begin{array}{l}\text { HEJ, } \\
\text { HFP, } \\
\text { MOJ, } \\
\text { MDJ, } \\
\text { OET, } \\
\text { OLT, }\end{array}$ & $\begin{array}{l}\text { AWX, } \\
\text { AWX, } \\
\text { IMY, } \\
\text { IMY, } \\
\text { HCS, } \\
\text { HCS, }\end{array}$ & $\begin{array}{l}\text { IOX, } \\
\text { IOX, } \\
\text { UXY, } \\
\text { UXY, } \\
\text { NOS, } \\
\text { NOS, }\end{array}$ & $\begin{array}{l}\text { SWO, } \\
\text { SWO, } \\
\text { BFZ, } \\
\text { BFZ, } \\
\text { YGW, } \\
\text { YGW, }\end{array}$ & $\begin{array}{l}\text { SYP, } \\
\text { SYP, } \\
\text { BML, } \\
\text { BML, } \\
\text { YUQ, } \\
\text { YUQ, }\end{array}$ & $\begin{array}{l}\text { NEL, } \\
\text { NFP, } \\
\text { WOJ, } \\
\text { WDJ, } \\
\text { REN, } \\
\text { RLN, }\end{array}$ & $\begin{array}{l}\text { DEQ, } \\
\text { DFQ, } \\
\text { NOG, } \\
\text { NDG, } \\
\text { GEW, } \\
\text { GLW, }\end{array}$ & $\begin{array}{l}\text { ZCX, } \\
\text { ZCX, } \\
\text { TXY, } \\
\text { TXY, } \\
\text { MDS, } \\
\text { MDS, }\end{array}$ & $\begin{array}{l}\text { JUX } \\
\text { JUX } \\
\text { ZTY } \\
\text { ZTY } \\
\text { LQS } \\
\text { LQS }\end{array}$ \\
\hline $\begin{array}{l}\text { SEX-SFX } \\
\text { BOY-BDY } \\
\text { YES-YLS }\end{array}$ & $\begin{array}{l}\text { TPD, } \\
\text { WLC, } \\
\text { CVM, }\end{array}$ & $\begin{array}{l}\text { MUR, } \\
\text { IKQ, } \\
\text { OWX, }\end{array}$ & $\begin{array}{l}\text { IKB, } \\
\text { MXG, } \\
\text { RGU, }\end{array}$ & $\begin{array}{l}\text { Low-S } \\
\text { HWJ, } \\
\text { VIS, } \\
\text { BJI, }\end{array}$ & $\begin{array}{l}\text { nilarity-1 } \\
\text { AGQ, } \\
\text { UNJ, } \\
\text { GOH, }\end{array}$ & $\begin{array}{l}\text { Ionword } \\
\text { VML, } \\
\text { XFH, } \\
\text { QMK, }\end{array}$ & $\begin{array}{l}\text { Backgrou } \\
\text { UOL, } \\
\text { MIU, } \\
\text { QNC, }\end{array}$ & $\begin{array}{l}\text { WUB, } \\
\text { VLA, } \\
\text { DMU, }\end{array}$ & $\begin{array}{l}\text { JNQ, } \\
\text { HFX, } \\
\text { GDV, }\end{array}$ & $\begin{array}{l}\text { ZVJ, } \\
\text { VRI, } \\
\text { VOT, }\end{array}$ & $\begin{array}{l}\text { PAI, } \\
\text { NMQ, } \\
\text { BVJ, }\end{array}$ & $\begin{array}{l}\text { LMU } \\
\text { GMZ } \\
\text { KWO }\end{array}$ \\
\hline \multicolumn{13}{|c|}{ Low-Similarity-Word Background } \\
\hline $\begin{array}{l}\text { SEX-SFX } \\
\text { BOY-BDY } \\
\text { YES-YLS }\end{array}$ & $\begin{array}{l}\text { CAT, } \\
\text { LEG, } \\
\text { BIT, }\end{array}$ & $\begin{array}{l}\text { HIM, } \\
\text { MAN, } \\
\text { AND, }\end{array}$ & $\begin{array}{l}\text { WAR, } \\
\text { GET, } \\
\text { HUG, }\end{array}$ & $\begin{array}{l}\text { RAT, } \\
\text { ASK, } \\
\text { AID, }\end{array}$ & $\begin{array}{l}\text { PIG, } \\
\text { HIM, } \\
\text { NOW, }\end{array}$ & $\begin{array}{l}\text { JAR, } \\
\text { JUG, } \\
\text { ROD, }\end{array}$ & $\begin{array}{l}\text { BAG, } \\
\text { ACT, } \\
\text { DIG, }\end{array}$ & $\begin{array}{l}\text { MAN, } \\
\text { SIN, } \\
\text { TOP, }\end{array}$ & $\begin{array}{l}\text { AND, } \\
\text { FAR, } \\
\text { CAT, }\end{array}$ & $\begin{array}{l}\text { HIT, } \\
\text { TUG, } \\
\text { BOG, }\end{array}$ & $\begin{array}{l}\text { OLD, } \\
\text { WAS, } \\
\text { OLD, }\end{array}$ & $\begin{array}{l}\text { BOY } \\
\text { EAT } \\
\text { JAM }\end{array}$ \\
\hline
\end{tabular}

APPENDIX B

Stimulus Materials Used in Experiment 2

\begin{tabular}{|c|c|c|c|c|c|c|c|c|c|c|c|c|}
\hline \multirow[t]{2}{*}{ Target } & \multicolumn{12}{|c|}{ Distractors } \\
\hline & \multicolumn{12}{|c|}{ Word Background } \\
\hline $\begin{array}{l}\text { SEX-SFX } \\
\text { DOG-DCG } \\
\text { THE-THL }\end{array}$ & $\begin{array}{l}\text { CAT, } \\
\text { MEN, } \\
\text { CAR, }\end{array}$ & $\begin{array}{l}\text { HIM, } \\
\text { SIT, } \\
\text { CRY, }\end{array}$ & $\begin{array}{l}\text { WAR, } \\
\text { LET, } \\
\text { SAD, }\end{array}$ & $\begin{array}{l}\text { RAT, } \\
\text { THE, } \\
\text { SON, }\end{array}$ & $\begin{array}{l}\text { PIG, } \\
\text { TAP, } \\
\text { BAR, }\end{array}$ & $\begin{array}{l}\text { JAR, } \\
\text { YES, } \\
\text { ANY, }\end{array}$ & $\begin{array}{l}\text { BAG, } \\
\text { KEY, } \\
\text { MUD, }\end{array}$ & $\begin{array}{l}\text { MAN, } \\
\text { HIM, } \\
\text { DUG, }\end{array}$ & $\begin{array}{l}\text { AND, } \\
\text { FAT, } \\
\text { WIN, }\end{array}$ & $\begin{array}{l}\text { HIT, } \\
\text { EAT, } \\
\text { WAY, }\end{array}$ & $\begin{array}{l}\text { OLD, } \\
\text { ART, } \\
\text { MAN, }\end{array}$ & $\begin{array}{l}\text { BOY } \\
\text { FUN } \\
\text { YOU }\end{array}$ \\
\hline \multicolumn{13}{|c|}{ Nonword Background } \\
\hline $\begin{array}{l}\text { SEX-SFX } \\
\text { DOG-DCG } \\
\text { THE-THL }\end{array}$ & $\begin{array}{l}\text { TCA, } \\
\text { NME, } \\
\text { RAC, }\end{array}$ & $\begin{array}{l}\text { MIH, } \\
\text { TSI, } \\
\text { YCR, }\end{array}$ & $\begin{array}{l}\text { RWA, } \\
\text { ELT, } \\
\text { DSA, }\end{array}$ & $\begin{array}{l}\text { TRA, } \\
\text { HTE, } \\
\text { NSO, }\end{array}$ & $\begin{array}{l}\text { GIP, } \\
\text { TPA, } \\
\text { RBA, }\end{array}$ & $\begin{array}{l}\text { RJA, } \\
\text { SYE, } \\
\text { NYA, }\end{array}$ & $\begin{array}{l}\text { GBA, } \\
\text { YKE, } \\
\text { UMD, }\end{array}$ & $\begin{array}{l}\text { AMN, } \\
\text { MHI, } \\
\text { GDU, }\end{array}$ & $\begin{array}{l}\text { NDA, } \\
\text { FTA, } \\
\text { NIW, }\end{array}$ & $\begin{array}{l}\text { HTI, } \\
\text { TAE, } \\
\text { YWA, }\end{array}$ & $\begin{array}{l}\text { DLO, } \\
\text { RTA, } \\
\text { NMA, }\end{array}$ & $\begin{array}{l}\text { YBO } \\
\text { NFU } \\
\text { UOY }\end{array}$ \\
\hline
\end{tabular}

